\title{
Use of radon barriers to reach an acceptable radon level
}

\author{
Torben Valdbjørn Rasmussen ${ }^{1, *}$ and Thomas Cornelius ${ }^{1}$ \\ ${ }^{1}$ Department of Building Technology and Process, BUILD, Aalborg University, A.C. Meyers Vænge 15, 2450 Copenhagen S, Denmark
}

\begin{abstract}
A method is presented for theoretically estimating the necessary airtightness of a radon barrier. Radon barriers are used to balance the indoor radon concentration. To balance radon at an acceptable level, for a given ventilation rate for indoor air, a barrier must fulfil the requirements for airtightness and the indoor-air radon penetration from the soil, which is determined by the radon concentration in the soil gas. The method identifies the optimal radon barrier for a building. Ten different radon barriers are evaluated. Barriers include system solutions based on materials such as bitumen-based radon blockers, wet-room membranes, reinforced fix mortar pastes, and polyethylene membranes. The barriers are tested using a modified version of the test method NBI 167/02 radon membrane: test of airtightness. The radon barriers are evaluated for a typical building construction for a single-family house with radon exposure from the ground. An acceptable radon concentration of $100 \mathrm{~Bq} / \mathrm{m}^{3}$ in indoor air is used in combination with a number of higher radon levels. The different radon barriers are evaluated in accordance with their ability to prevent air penetration from the ground. Furthermore, how mounting a barrier can affect the durability of a building is discussed, as the measures may create a far more vulnerable building.
\end{abstract}

Keywords: Radon, barrier, penetration, model, indoor air

\section{Introduction}

Radon-222 develops from the radioactive decay of radium-226 and has a half-life of 3.8 days. This gas seeps through the soil into buildings, and if not diluted, with outdoor air, by ventilation, much higher exposure levels can occur indoors, where human exposure occurs, than outdoors [1] [2]. Thus, radon affects occupants through the indoor climate.

The World Health Organization recommends that states introduce requirements for the maximum concentration of radiation from natural sources in indoor air. These recommendations are the result of the determination by the World Health Organization of radon being responsible for $3 \%$ to $14 \%$ of lung cancer cases, depending on the average radon exposure in different countries [3]. The results indicate that radon is the second-leading cause of lung cancer (smoking tobacco is still the primary cause). Therefore, it is crucial to prevent radon from penetrating buildings. Since 2010, the Danish Building Regulations have required that buildings must be constructed to ensure that indoor radon levels remain below $100 \mathrm{~Bq} / \mathrm{m}^{3}$ [4].

One way to prevent radon from penetrating buildings is to establish a radon barrier facing the ground because air penetration from the ground underneath a building is the main source of radon in indoor air [5]. Because an air barrier can prevent radon from penetrating buildings, it is important to determine the airtightness of such a barrier when used as a radon barrier. Moreover, the barrier must be sufficiently airtight and have airtight joints at the corners, across floor-level changes, around barrier-penetrating pipes, and against floor drains.

The paper presents a method for estimating the theoretically optimal radon barrier for a building. An optimal radon barrier provides an acceptable indoor radon concentration with an acceptable change in building physics. The use of a barrier to prevent radon penetration from the soil often causes a change in the building physics related to the moisture level in building materials from rising moisture from the soil.

Ideally, the indoor radon concentration is lowered to a level that meets the requirements of the National Building Regulations. However, for existing buildings, a higher indoor radon concentration might be considered acceptable, considering the costs of preventing a rise in the moisture level from the soil. Therefore, a barrier must be evaluated in accordance with its ability to prevent soil gas penetration from the ground and its influence on the overall moisture level in affected building materials.

The paper evaluates 10 different radon barriers, which were tested as single-system solutions. The barriers include system solutions based on materials such as bitumen-based radon blockers, wet-room membranes, reinforced fix mortar pastes, and mortar and polyethylene membranes.

\footnotetext{
$\bar{*}$ Corresponding author: tvr@sbi.aau.dk
} 
The barriers were tested using a modified version of the NBI 167/02 radon membrane: test of airtightness test method [6], which was used for determining the airtightness of a radon barrier used as a system solution. The test method was modified by providing a digital stirring and control system and by introducing equipment to determine the overall mean air-pressure difference over the barrier.

The paper presents an evaluation of the 10 radon barriers used as system solutions based on the results obtained using the improved test method. The evaluation of the radon barriers was based on a model representing a typical building construction for a single-family house, with radon exposure from the ground, which varies from less than 5,000 to $150,000 \mathrm{~Bq} / \mathrm{m}^{3}$ in the soil gas. An acceptable concentration of radon of $100 \mathrm{~Bq} / \mathrm{m}^{3}$ in indoor air was used. However, several higher radon levels in indoor air were included in the paper as acceptable levels, identifying an optimal radon barrier for a single-family house.

\section{Theoretical background}

A house with the ground area denoted as $A$, ceiling height $h$, and air-change rate $q$, where indoor air is diluted with outdoor air with a radon content of $r$ and the indoor-air radon content is denoted as $R$, provides the theoretical basis (Figure 1). Assuming soil gas is the source that increases the indoor-air radon concentration, the maximum penetration of soil gas with radon content (denoted as $R_{g}$ ) to maintain an acceptable indoor radon concentration was found by use of the equilibrium equation.

Assuming the air pressure difference between the interior and exterior of the building is constant over time the equilibrium equation can be used to describe the static equilibrium of all internal and external forces of the system [7]. In the static case, the equilibrium equation is;

$$
K * u=F
$$

Where, $K$ is the stiffness matrix of the system, $u$ is the vector with the nodal displacements and $F$ represents the external forces.

The equilibrium equation is used to describe the equilibrium between the constant radon concentration in the indoor air and the supply of radon from the soil gas and the exterior air. The soil gas and the exterior air are both assumed to have a constant but different radon concentration. The equilibrium is given by Equation (2).

$$
q * A * h * R=y * r+x * R_{g}
$$

The radon content in the air outlet equals the sum of radon from the air supply.

The equilibrium equation is also used to describe the equilibrium between the volume of indoor air ventilated out of the building and the volume of air supply needed from the soil gas and the exterior air, not to change the air pressure difference over the building envelope. The equilibrium is given by Equation (3)

$$
y+x=q^{*} A * h
$$

The variables $x$ and $y$ are the only undefined in these equations, equation (1) and (2).

The air supply provided by the ventilation and penetrating air from the soil equals the air outlet of the building.

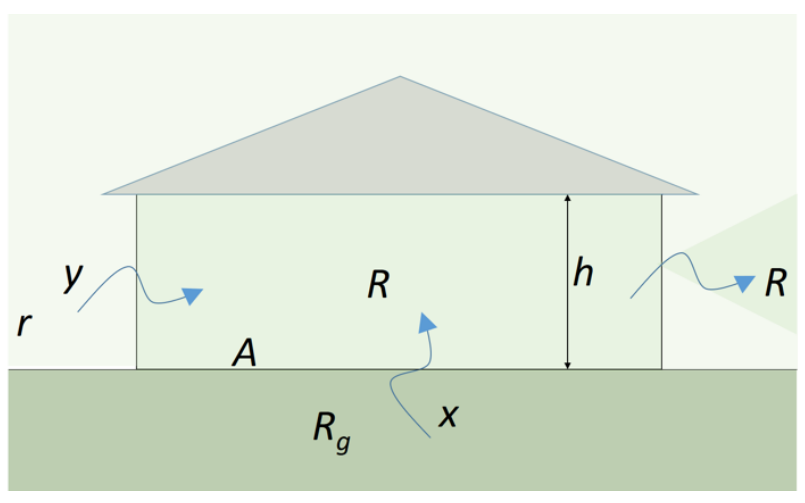

Fig. 1. The theoretical basis for a single-family house where the air pressure difference between the interior and exterior is constant over time.

\section{Theory to practice}

From the theoretical background, the requirements for the penetration of radon from soil gas can be measured. For a single-family house, the maximum penetration of soil gas to maintain an acceptable indoor radon concentration of $100 \mathrm{~Bq} / \mathrm{m}^{3}$ was found for a number of radon exposures from soil gas. The radon concentration in soil gas varied from less than 1,000 to $150,000 \mathrm{~Bq} / \mathrm{m}^{3}$. The ground area of the house was $100 \mathrm{~m}^{2}$ with a ceiling height of $2.5 \mathrm{~m}$. To maintain an acceptable indoor environment, the air-change rate was $0.5 \mathrm{~h}^{-1}$, which is equivalent to changing all of the indoor air every 2 hours.

Indoor air was diluted with outdoor air, with a radon content of $5 \mathrm{~Bq} / \mathrm{m}^{3}$. To meet and maintain an acceptable indoor radon concentration of $100 \mathrm{~Bq} / \mathrm{m}^{3}$ the requirements for the penetration of soil gas containing radon is shown in Figure 2. Additionally, Figure 2 illustrates how the penetration of soil gas can increase when to meet and maintain an acceptable indoor radon concentration of $300 \mathrm{~Bq} / \mathrm{m}^{3}$ and $600 \mathrm{~Bq} / \mathrm{m}^{3}$. Penetration was calculated in litres per minute. 


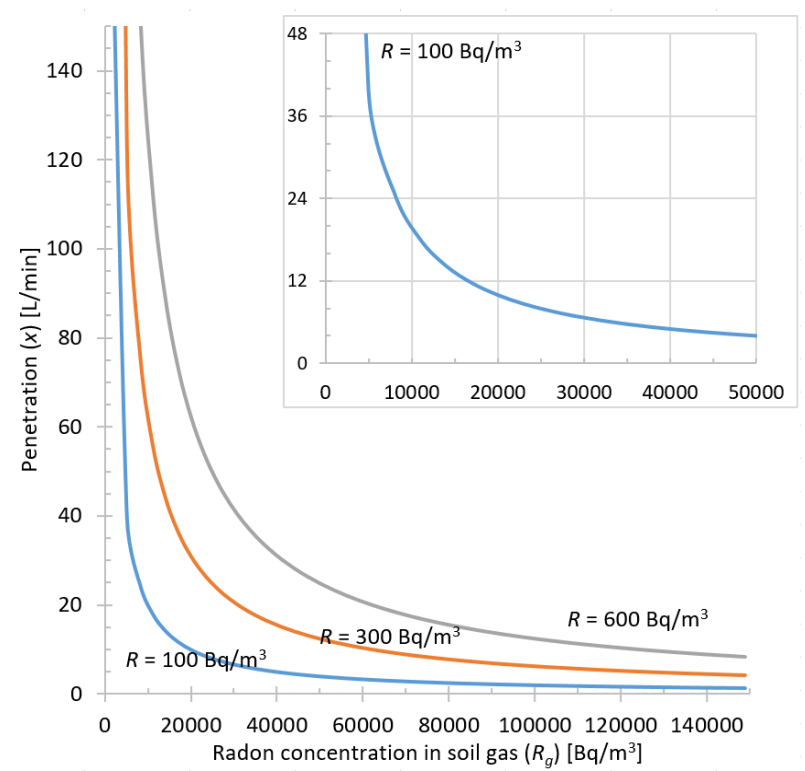

Fig. 2. Allowed penetration of soil gas with a given radon concentration, reaching an acceptable radon concentration in indoor air of $100 \mathrm{~Bq} / \mathrm{m}^{3}, 300 \mathrm{~Bq} / \mathrm{m}^{3}$, and $600 \mathrm{~Bq} / \mathrm{m}^{3}$. Indoor air was diluted with outdoor air with a radon concentration of $5 \mathrm{~Bq} / \mathrm{m}^{3}$ and an air-change rate of $0.5 \mathrm{~h}^{-1}$. Soil gas penetrates indoor air through the ground slab.

\section{Air Penetration rates}

The radon barriers must fulfil the requirements for air penetration related to the radon concentration in soil gas. The barriers used as system solutions were tested using the modified version of the NBI 167/02 radon membrane: test of airtightness test method [6], which determined the airtightness of a radon barrier used as a system solution.

\subsection{Barriers}

Ten types of barriers were tested as system solutions, which are denoted as Systems A through J, as follows:

- System A: fix mortar paste combined with acrylic sealant, also used as a wet-room membrane;

- System B: firm bitumen-based radon blocker combined with a two-component floating sealant;

- System C: a reinforced fix mortar paste combined with acrylic sealant;

- System D: one-component floating membrane combined with edge reinforcements, epoxy, and elastic pipe collars;

- System E: two-component fix mortar paste combined with edge reinforcements, epoxy, and elastic pipe collars;

- System F: foil system consisting of nonwovens filled with a two-component fix mortar paste combined with edge reinforcements, epoxy, and elastic pipe collars;

- System G: polyethylene membrane with solid tape joints, acrylic primer, and elastic pipe collars;
- System H: polyethylene membrane with solid tape joints, acrylic adhesive, acrylic primer, and elastic pipe collars;

- System I: noise-reducing aluminium foil-coated subflooring with aluminium butyl tape joints, primer, and elastic pipe collars;

- System J: noise-reducing aluminium foil-coated subflooring with aluminium butyl tape joints, primer, elastic pipe collars, and a one-component flow membrane.

Mounting the test material denoted System B inside the mock-up is shown in Figure 3. System B is the firm bitumen-based radon blocker combined with a twocomponent floating sealant. Mounting the twocomponent fix mortar paste combined with edge reinforcements, epoxy, and elastic pipe collars denoted System E is shown in Figure 4.
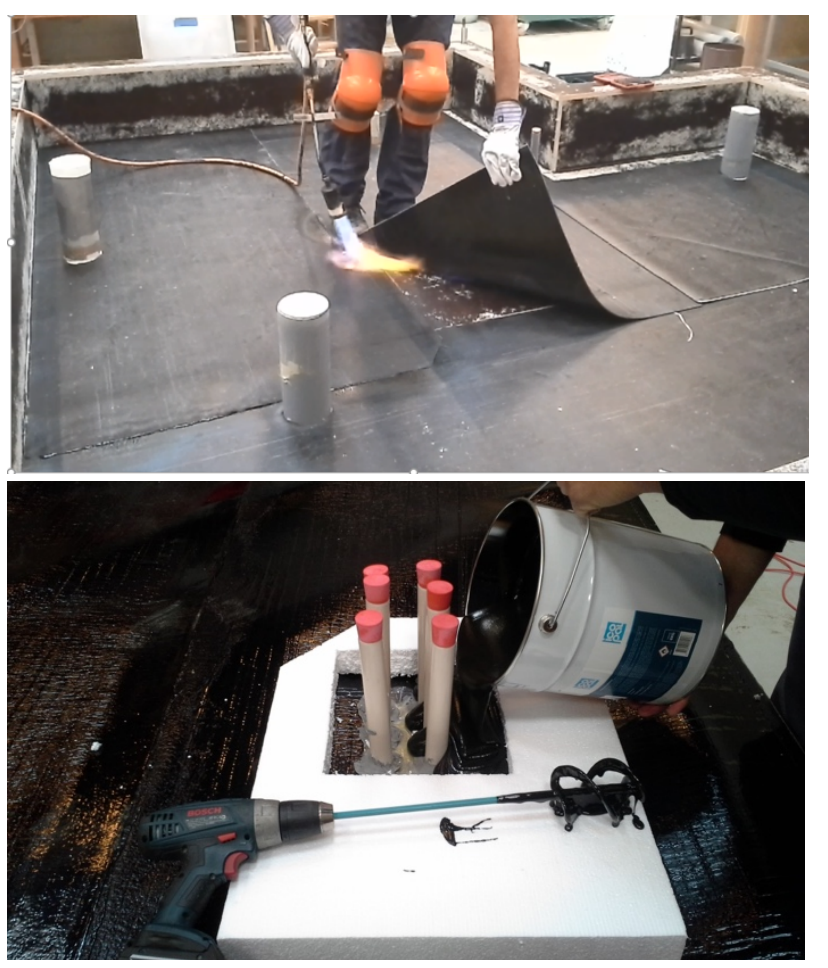

Fig. 3. Mounting the test material System B inside the mockup. System B is the firm bitumen-based radon blocker (upper picture) combined with a two-component floating sealant (lower picture).

The barriers were used as delivered, and the manufacturer mounted them inside the mock-up. The tests were to start 40 hours after mounting the barrier to ensure a stress-free barrier and joints. The tests set no specific requirements for the indoor climate at the laboratory used for testing; however, the laboratory climate should be a dry tempered room with a temperature ranging between $17^{\circ} \mathrm{C}$ and $25^{\circ} \mathrm{C}$ and with relative humidity ranging between $15 \%$ and $65 \%$. However, shorter periods with a temperature and relative humidity outside these intervals were accepted. 


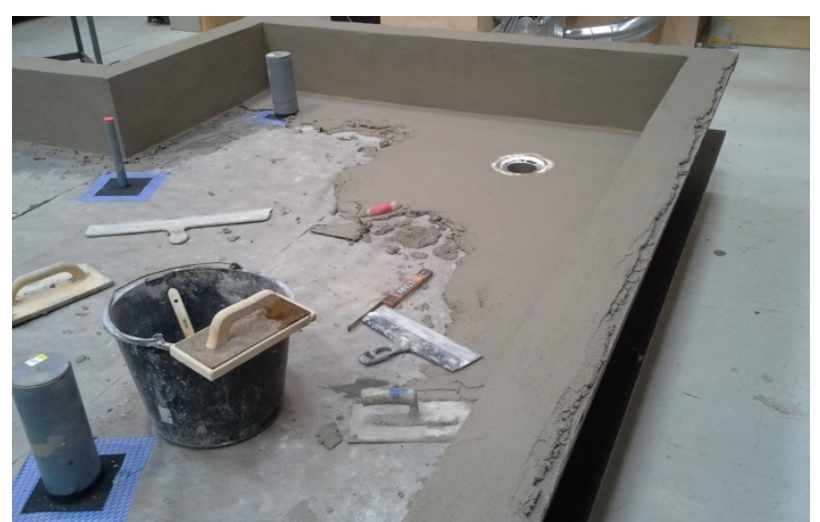

Fig. 4. Mounting the test material System E inside the mockup. System E is the two-component fix mortar paste combined with edge reinforcements, epoxy, and elastic pipe collars.

\subsection{Test of penetration rates}

The test determines the air penetration through a material tested for suitability as a radon barrier. The test evaluates how well a barrier prevents soil gas with radon from penetrating indoor air. The barrier was mounted inside a mock-up providing a stable basis with penetrating pipes, an elevation, and narrow-angled and wide-angled corners. The test method was an upgrade of the test method NBI 167/02 [8]. The airtightness of a barrier was determined as the air penetration through the barrier and its joints for a difference in air pressure over the barrier of $30 \mathrm{~Pa}$, denoted as $q_{30}$. The difference in air pressure over the barrier was the difference in the air pressure between the air inside the volume of the mock-up, designed as a box, and the air in the surrounding test laboratory.

\subsection{Measurement Set-Up}

The test was conducted by mounting the test material inside a mock-up. After moulding the test material, the mock-up was filled with pressure-firm thermal insulation of mineral wool. On top of the firm insulation, a layer of the test material was mounted to seal the volume of the mock-up that holds the firm insulation enveloped by the test material. The constant airflow drawn from the sealed volume of the mock-up was measured. The airflow provides a constant air-pressure difference between the air inside the mock-up and the outside air in the surrounding test laboratory where the test was conducted.

\subsection{Equipment}

The barrier was mounted in a mock-up made of laminated wooden boards $3.0 \mathrm{~m}$ in length and width and $0.3 \mathrm{~m}$ high with a notch of 1.0 by $1.0 \mathrm{~m}$, with changed floor levels, penetrating pipes, and floor drains, as shown in Figure 5. Using a fan, the air was extracted out of the volume, consisting of the pressure-firm thermal insulation material enveloped by the test barrier material. The coherent values of the airflow and the difference in air pressure between the air inside the volume of the mock-up and the air in the surrounding test laboratory was measured and logged systematically.

Using the PC program TECLOC3 from BlowerDoor Gmbh, the data were logged by connecting the PC to a unit measuring the pressure difference and the fan. The fan was a Minneapolis micro leakage meter, type FD E51-767, which measured the airflow in the range between 0.09 and $79 \mathrm{~m}^{3} / \mathrm{h}$. The fan was mounted on a disc with a circular hole to measure the airflow. Individual discs were mounted, and each had a circular hole of $3.8,8.0,20$, or $45 \mathrm{~mm}$. A PC controls the fan to extract the air out of the volume of the mock-up, introducing a predetermined difference in air pressure between the volume within the mock-up and the air in the surrounding test laboratory. The airflow was measured for a number of predetermined differences in the air pressure between the volume within the mock-up and the air in the surrounding test laboratory.

As the air pressure within the mock-up was not homogeneously distributed, five air-pressure difference measurement units were mounted on the top layer of the test material. The mean value of the pressure difference between the volume within the mock-up and the air in the surrounding test laboratory was determined from the five units and was used to calibrate the pressure for the airflow measurements.

To measure the airtightness of the barriers with low airflow and in the lower ranges of the capacity of the micro leakage meter, it was necessary to add air infiltration through well-defined openings. The welldefined openings were added using discs with a diameter of $7,10,14$, or $20 \mathrm{~mm}$. The airflow through the welldefined opening was subtracted from the measured airflow.

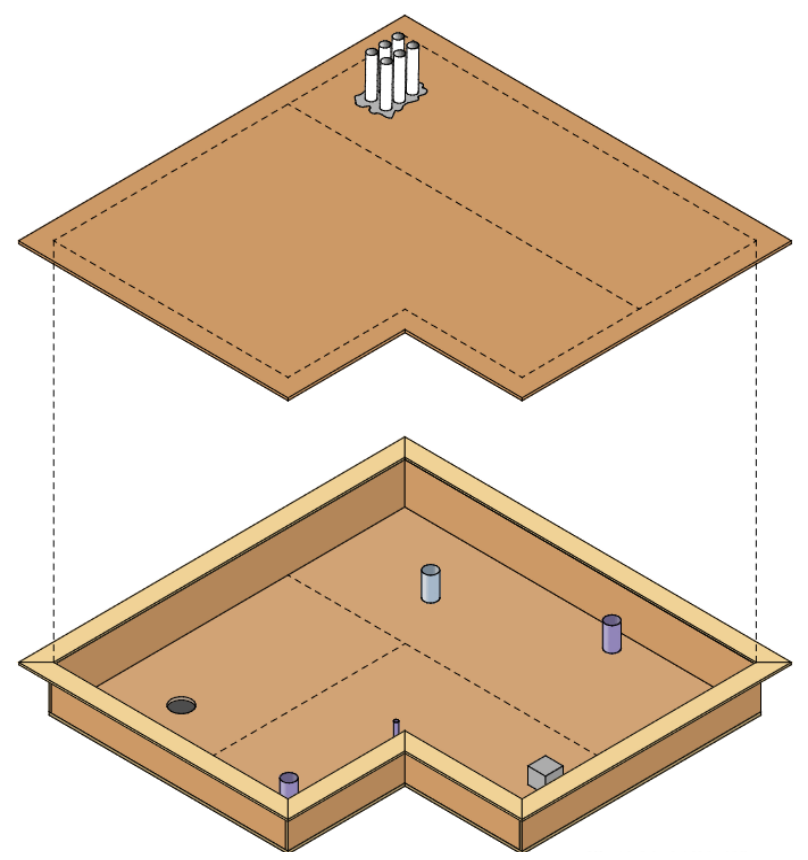

Fig. 5. Mock-up used for measuring the airtightness, measured as the air penetration. 


\subsection{Processing the results}

Mounted over the barrier system, the airflow was measured at four different air-pressure levels of 30, 50, 70 , and $90 \mathrm{~Pa}$ controlled by the air-pressure measuring equipment. At each pressure level, four measurements were performed using four different well-defined openings. For all 16 measurements, the areas of the welldefined opening, the individual air-pressure difference in the five air-pressure difference measurement units, and the airflow through the suction point were measured. The measurements were used to calculate the airflow in litres per minute $[\mathrm{L} / \mathrm{min}]$ for a $30-\mathrm{Pa}$ mean pressure difference, denoted as $q_{30}$, over the barrier system, where $q_{30}$ was determined for the individual barrier systems.

The airflow for a 30-Pa mean pressure difference over the barrier system comprises the soil gas penetration for a one-floor building with a ground area of $100 \mathrm{~m}^{2}$ with a difference in air pressure over the building envelope of 1 to $4 \mathrm{~Pa}[4,5,6,8]$. The highest allowed penetrations of soil gas with a radon concentration not exceeding an acceptable level in indoor air of $100 \mathrm{~Bq} / \mathrm{m}^{3}, 300 \mathrm{~Bq} / \mathrm{m}^{3}$, and $600 \mathrm{~Bq} / \mathrm{m}^{3}$ were determined. Soil gas penetration was found as the intersection between the air balance indoors, given by the air change and an acceptable radon concentration from Equations (2) and (3), and the penetration of soil gas, $q_{30}$. For the calculations, indoor air was assumed to be diluted with outdoor air with a radon concentration of $5 \mathrm{~Bq} / \mathrm{m}^{3}$ [9]. Additionally, the air-change rate in the building was set at $0.5 \mathrm{~h}^{-1}$ [10]. Furthermore, it was assumed that the soil gas was the source of radon in indoor air and that radon penetrates from the ground through the slab $[4,5,6,8]$.

Figure 6 shows the determination of soil gas with a radon concentration not exceeding acceptable levels in indoor air of $100 \mathrm{~Bq} / \mathrm{m}^{3}, 300 \mathrm{~Bq} / \mathrm{m}^{3}$, and $600 \mathrm{~Bq} / \mathrm{m}^{3}$ for barrier System C.

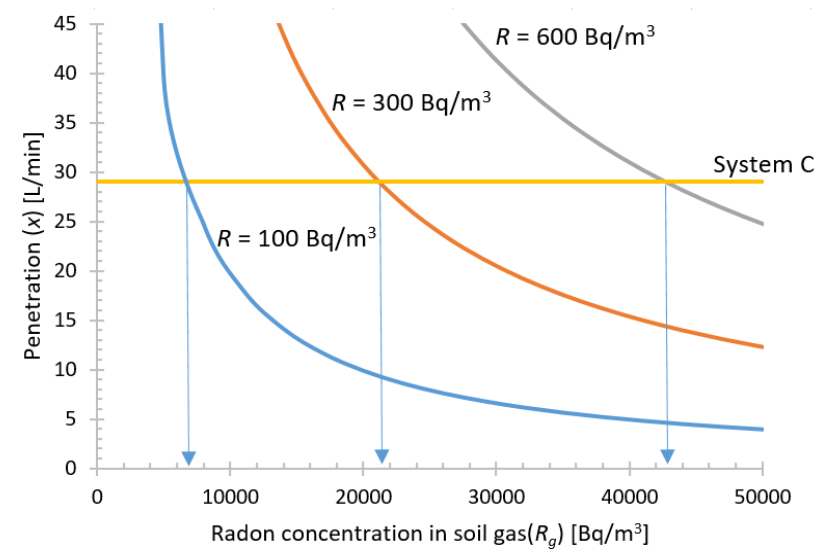

Fig. 6. Radon barrier System $C$ allows the penetration of soil gas with a radon concentration of $7,000 \mathrm{~Bq} / \mathrm{m}^{3}$, reaching an acceptable level of $100 \mathrm{~Bq} / \mathrm{m}^{3}$. Indoor air was diluted with outdoor air with a radon concentration of $5 \mathrm{~Bq} / \mathrm{m}^{3}$, and the airchange rate was $0.5 \mathrm{~h}^{-1}$. The penetration of soil gas with a radon concentration of $21,500 \mathrm{~Bq} / \mathrm{m}^{3}$ reached an acceptable level of $300 \mathrm{~Bq} / \mathrm{m}^{3}$, and soil gas with a radon concentration of $43,000 \mathrm{~Bq} / \mathrm{m}^{3}$ reached a level of $600 \mathrm{~Bq} / \mathrm{m}^{3}$ in indoor air.

\section{Results}

To reach an acceptable radon concentration in indoor air, the penetration rates and determined radon concentration in soil gas are not to exceed $100 \mathrm{~Bq} / \mathrm{m}^{3}, 300 \mathrm{~Bq} / \mathrm{m}^{3}$, and $600 \mathrm{~Bq} / \mathrm{m}^{3}$, which are shown in Table 1.

Table 1. The maximum penetration, of soil gas with radon content $\left(R_{\mathrm{g}}\right)$, through the ground slab to reach indoor air with a radon concentration of $100 \mathrm{~Bq} / \mathrm{m}^{3}, 300 \mathrm{~Bq} / \mathrm{m}^{3}$, and $600 \mathrm{~Bq} / \mathrm{m}^{3}$

for each tested radon barrier system. How well a barrier prevents soil gas from penetrating indoor air is defined as the airflow penetration rate, $q_{30}$.

\begin{tabular}{|c|c|c|c|c|}
\hline \multirow[b]{2}{*}{$\begin{array}{l}\text { Barrier } \\
\text { system }\end{array}$} & \multirow{2}{*}{$\begin{array}{c}\text { Airflow, } \\
\text { penetra- } \\
\text { tion rate } \\
q_{30} \\
{[\mathrm{~L} / \mathrm{min}]}\end{array}$} & \multicolumn{3}{|c|}{$R_{g}$ in soil gas } \\
\hline & & $\begin{array}{c}100 \\
{\left[\mathrm{~Bq} / \mathrm{m}^{3}\right]}\end{array}$ & $\begin{array}{c}300 \\
{\left[\mathrm{~Bq} / \mathrm{m}^{3}\right]}\end{array}$ & $\begin{array}{c}600 \\
{\left[\mathrm{~Bq} / \mathrm{m}^{3}\right]}\end{array}$ \\
\hline A & 12 & 16,000 & 70,000 & 140,000 \\
\hline B & 1.9 & 120,000 & 330,000 & 650,000 \\
\hline $\mathrm{C}$ & 29 & 7,000 & 21,500 & 43,000 \\
\hline $\mathrm{D}$ & 4.8 & 40,000 & 130,000 & 260,000 \\
\hline $\mathrm{E}$ & 4.7 & 42,500 & 132,500 & 265,000 \\
\hline $\mathrm{F}$ & 12.6 & 16,000 & 49,000 & 98,000 \\
\hline G & 132 & 3,000 & 5,000 & 10,000 \\
\hline $\mathrm{H}$ & 8.9 & 22,000 & 69,000 & 140,000 \\
\hline I & 63.9 & 4,500 & 10,000 & 20,000 \\
\hline $\mathrm{J}$ & 16.6 & 12,000 & 37,000 & 75,000 \\
\hline
\end{tabular}

\section{Control of radon indoor}

An efficient way to avoid radon infiltrating a building and to control the radon concentration in indoor air is to combine the three design criteria:

1) A radon barrier:

a) an airtight concrete slab or

b) a radon barrier placed in or underneath the ground slab;

2) Lowering the air pressure at the lower zone of the ground slab;

3) Effective dilution of indoor air with outdoor air, see Figure 7.

In this way, the radon concentration in indoor air can be controlled and maintained at an acceptable level, even if failures occur in the radon barrier [11]. However, in contrast to new buildings, for buildings that are already constructed, the three design criteria may not be possible to implement. 


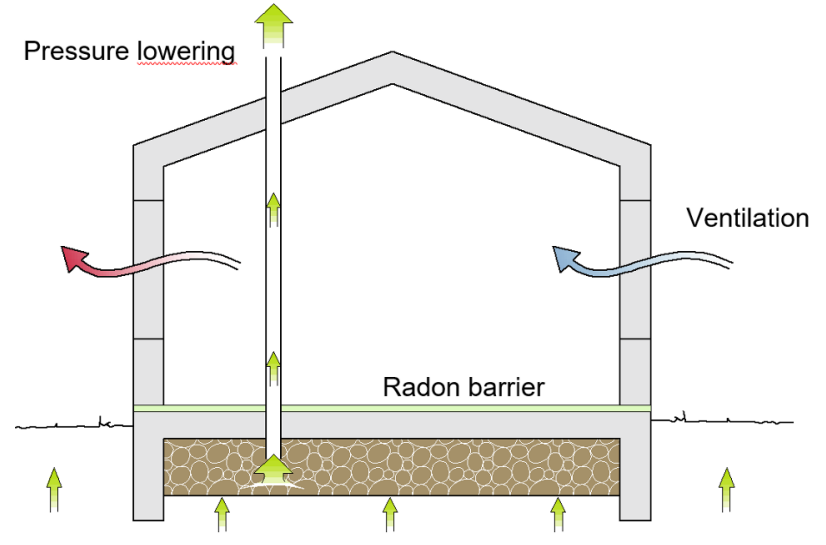

Fig. 7. By combining the three design criteria, the radon penetration and concentration in indoor air can be controlled by 1) establishing a barrier that prevents soil gas from penetrating from the ground, 2) lowering the air pressure in the lower zone of the ground slab, and 3) diluting indoor air with outdoor air.

\section{Moisture challenges}

During building construction, a radon barrier can easily be applied, creating a radon barrier as an airtight barrier within or above the ground slab or in the ground below the slab. This can be done in various ways with suitable fixation onto the materials and surfaces, in combination with the moisture barrier that prevents moisture from the ground from reaching constructions above the foundation or the interior of a basement. Applying a radon barrier to a building already constructed can affect the durability of the building, especially for a heritage building, because measures may create a far more vulnerable building and change its robustness to withstand moisture and user behaviour.

When deciding on a radon barrier to be mounted on the ground slab or basement wall and floor, the influence and change in moisture load and content of other building components and constructions must be considered. This means that the changed water vapour diffusion and resulting rise in soil moisture load may create a more vulnerable building after mounting a radon barrier for the construction. Special attention must be focused on the risk of mould growth, for example, in an air cavity behind a radon barrier that is not bonded to the underlayment.

By diffusion radon can penetrate through the ground slab or basement wall and floor. The ability for gases, vapour and other minor molecules to penetrate through the ground slab, basement wall and floor by diffusion depends on the individually permeability of the ground slab, basement wall and floor.

Diffusion through concrete is considered limited. Fix mortar paste is considered to be able to reduce diffusion but not to prevent penetration by diffusion. As the ability to limit diffusion is related to the density of the fix mortar paste and the thickness of the mortar paste layer. Even miner cracks can increase the diffusion [12].

Investigations of radon diffusion through a normal concrete slab $150 \mathrm{~mm}$ in thickness without cracks have shown to contribute to radon indoor by approximately $15-20 \mathrm{~Bq} / \mathrm{m}^{3}$. For the investigations an air-change rate of $0.5 \mathrm{~h}^{-1}$ was provided in the building as well as a radon content in the soil gas of $500,000 \mathrm{~Bq} / \mathrm{m}^{3}$ [13].

In Denmark the general radon content in the soil gas is substantial lower, approximately $50,000 \mathrm{~Bq} / \mathrm{m}^{3}$ [14]. In this case the contribution by diffusion of radon to the indoor air is substantially lower, approximately $2 \mathrm{~Bq} / \mathrm{m}^{3}$.

Radon penetration through the ground slab, basement wall and floor by diffusion in buildings today represent a limited contribution to the overall indoor-air radon content, which main source is the soil gas from the ground.

\section{Discussion}

Soil gas penetrating through the ground slab is the primary source of radon in indoor air in most countries, including Denmark [11]. Therefore, the geological composition of the ground on which a building is situated, radon concentration in soil gas, soil gas penetration through the ground slab, and air-change rate all set the level for the indoor radon concentration. Radon seeps into a building through soil gas penetration from the ground through cracks or other unforeseen openings in the ground construction [15]. Therefore, it is of great importance to control soil gas penetration e.g. using a radon barrier.

Establishing a barrier that prevents soil gas penetration from the ground is one of the efficient ways to avoid radon from penetrating a building and to control the radon concentration in indoor air. However, when combining the three mentioned design criteria [5], the radon concentration in indoor air can be controlled in a robust way and maintained at an acceptable level. If the air pressure in the lower zone of the ground slab cannot be lowered, the choice of a radon barrier is crucial for the indoor radon concentration.

The improved test method and presented theory aid in choosing a radon barrier to reach an acceptable indoor radon concentration. However, the change in the moisture level in the building components must be considered when choosing the most suitable radon barrier. Moreover, the most suitable radon barrier depends on individual building physics.

The requirements for the airtightness of a radon barrier, the penetration rate, $q_{30}$, can be found from the radon concentration in the soil gas in the ground underneath a building. In some cases, a diffusion-tight radon barrier can be used and in other cases, a diffusionopen barrier is preferred. The choice of barrier depends on the moisture level after mounting the radon barrier. It is important to choose a radon barrier that is sufficiently airtight so that it may constitute the needed radon barrier while contributing to the building physics.

From the theoretical processing of the test results, it was found that it is possible to choose a radon barrier based on the acceptable radon concentration in indoor air and the soil gas in the ground underneath a building. The 
theoretical processing of the test results show that the radon barrier System A, with the penetration rate $q_{30}$ of $12 \mathrm{~L} / \mathrm{min}$ can provide an acceptable radon concentration in indoor air that is less than or equal to $100 \mathrm{~Bq} / \mathrm{m}^{3}$ in a building on soil with a radon concentration less than or equal to $16,000 \mathrm{~Bq} / \mathrm{m}^{3}$. If the soil gas contains between $16,000 \mathrm{~Bq} / \mathrm{m}^{3}$ and $70,000 \mathrm{~Bq} / \mathrm{m}^{3}$, an acceptable indoor radon concentration of between $100 \mathrm{~Bq} / \mathrm{m}^{3}$ and 300 $\mathrm{Bq} / \mathrm{m}^{3}$ can be reached. For the theoretical processing, it was assumed that indoor air was diluted with outdoor air with a radon concentration of $5 \mathrm{~Bq} / \mathrm{m}^{3}$ at an air-change rate of $0.5 \mathrm{~h}^{-1}$.

From the evaluations of Systems G, H, I, and J, it was found that it is important to be aware of how the joints perform. This is because Systems G and H and Systems I and $\mathbf{J}$ are alike except for how the joints are performed.

\section{Conclusion}

A theoretical processing method of results gained from an improved test method for determining the airtightness of a radon barrier as a system solution was demonstrated. The improved test method is the NBI 167/02 method [6] developed by the Norwegian Building Research Institute.

The theoretical processing of the results determining the airtightness of the radon barriers as system solutions made it possible to choose a radon barrier from the choice of acceptable radon concentrations in indoor air and in soil gas in the ground underneath a building. However, the acceptable radon concentration in indoor air could be compromised because the choice of a suitable radon barrier depends on the change in moisture level in the building after mounting the radon barrier. A radon barrier must contribute to the building physics, creating a more robust building.

The theoretical processing method assumed that only soil gas and the atmosphere contain radon and that the soil gas was the source of radon in indoor air. However, materials used in buildings can contribute to the concentration of radon in indoor air if they contain radium and the chemical elements of uranium and thorium (e.g. granite and alum shale). The contribution to the concentration of radon in indoor air from building materials are rarely significant.

Acknowledgements: This study was supported by Realdania, which is a philanthropic association focusing on solving challenges in Danish society in cooperation with the government, municipalities, foundations, associations, private businesses, and local, voluntary enthusiasts.

\section{References}

1. W.W. Nazaroff, Radon transport from soil to air, Rev. Geophys. 30. 137-160 (1992)

2. B. Brunekreef and S.T. Holgate, Air pollution and health, The lancet. 360. 1233-1242 (2002)

3. H. Zeeb and F. Shannoun (eds.). WHO Handbook on indoor radon - A public health perspective. World Health Organization. Geneva. 94 (2009)

4. Danish Enterprise and Construction Authority. Danish Building Regulations 2010 (2010)

5. T.V. Rasmussen. Design criteria for achieving acceptable indoor radon concentration. In: International Journal of Civil, Environmental, Structural. Construction and Architectural Engineering. 10. 3. 268-273 (2016)

6. T.V. Rasmussen and T. Cornelius. Radon barrier: Method of testing airtightness - 2nd edition. 11th Nordic Symposium on Building Physics, NSB2017. 11-14 June. Trondheim. Norway (2017)

7. Y.K. Kyosev, The finite element method (FEM) and its application to textile technology, Simulation in Textile Technology (2012)

8. SINTEF Byggforsk. NBI 167/02, Radon membrane: Test of airtightness. February 2016. Norge: SINTEF Byggforsk. Located on: http://www.byggforsk.no

9. T.V. Rasmussen. SBi-instructions 270: Measuring radon in buildings; in Danish). Danish Building Research Institute, Aalborg University. Copenhagen. Denmark (2018)

10. Transport, Building and Housing Agency. Danish Building Regulations 2018 (2018)

11. T.V. Rasmussen. SBi-instructions 233 2nd ed.: Measures preventing radon to penetrate into new buildings. In Danish. Danish Building Research Institute, Aalborg University. Hørsholm. Denmark. 44 p. (2010)

12. Font Guiteras, Lluís. (1997). Radon generation, entry and accumulation indoors. Universitat Autònoma de Barcelona. Located on: http://www.tdr.cesca.es/TDX/TDX UAB/TESIS/A VAILABLE/TDX-0506108-225143//lfg1de2.pdf

13. Clavensjö, B., \& Åkerblom, G. (2004). Radonboken: Förebyggande åtgärder i nya byggnader. Stockholm: Formas.

14. Danish Health Authority, www.sst.dk

15. Danish Transport and Construction Agency. Radon and single-family houses. In Danish. Copenhagen. 15p. (2007) 